\title{
Casimir force in critical ternary polymer solutions
}

\author{
H.Ridouane, E.-K.Hachem, M.Benhamou* \\ Laboratoire de Physique des Polymères et Phénomènes Critiques, \\ Faculté des Sciences Ben M'sik, B.P. 7955, Casablanca, Morocco
}

Received November 24, 2003

\begin{abstract}
Consider a mixture of two incompatible polymers $A$ and $B$ in a common good solvent, confined between two parallel plates separated by a finite distance $L$. We assume that these plates strongly attract one of the two polymers close to the consolute point (critical adsorption). The plates then experience an effective force resulting from strong fluctuations of the composition. To simplify, we suppose that either plates have the same preference to attract one component (symmetric plates) or they have an opposed preference (asymmetric plates). The force is attractive for symmetric plates and repulsive for asymmetric ones. We first exactly compute the force using the blob model, and find that the attractive and repulsive forces decay similarly to $L^{-4}$. To go beyond the blob model that is a mean-field theory, and in order to get a correct induced force, we apply the Renormalization-Group to a $\varphi^{4}$-field theory ( $\varphi$ is the composition fluctuation), with two suitable boundary conditions at the surfaces. The main result is that the expected force is the sum of two contributions. The first one is the mean-field contribution decaying as $L^{-4}$, and the second one is the force deviation originating from strong fluctuations of the composition that decreases rather as $L^{-3}$. This implies the existence of some cross-over distance $L^{*} \sim a N \phi^{1 / 2}$ ( $a$ is the monomer size, $N$ is the polymerization degree of chains and $\phi$ is the monomer volumic fraction), which separates two distance-regimes. For small distances $\left(L<L^{*}\right)$, the mean-field force dominates, while for high distances $\left(L>L^{*}\right)$ the fluctuation force is more important.
\end{abstract}

Key words: ternary polymer solutions, confinement, Casimir force

PACS: $64.75 .+g, 68.45 .-v, 61.41 .+e$

\section{Introduction}

Consider a binary liquid made of two components $A$ and $B$ of different chemical nature, which is in contact with a solid wall. Close to the consolute point $T_{\mathrm{c}}$, one of the two components prefers to be condensed near the wall. This is the critical

*Author for correspondence; E-mail: m.benhamou@univh2m.ac.ma 
adsorption, which has been the subject of numerous theoretical and experimental studies $^{1}[1-15]$. Also, critical adsorption has been observed for a one-component fluid near the critical point, which is in contact with an attractive wall [16].

From a theoretical point of view, the first formulation is due to Fisher and de Gennes [7]. Using a scaling argument, the authors show, in particular, that far from the surface (distal regime) and at the critical point, the composition fluctuation profile of the preferred component, $\phi(z)$, decays with the distance $z$ from the wall according to a universal law, that is $\phi(z) \sim z^{-\beta_{\mathrm{t}} / \nu_{\mathrm{t}}}$. Here, $\beta_{\mathrm{t}} \simeq 0.325$ and $\nu_{\mathrm{t}} \simeq 0.63$ are the standard bulk critical exponents of Ising-like magnetic materials $[17,18]$. To take into account the effect of the wall, one has introduced a surface field $h$, which is proportional to the difference of surface chemical potentials, $\mu_{A}^{\mathrm{s}}$ and $\mu_{B}^{\mathrm{s}}$, of the two components, that is $h \sim\left(\mu_{A}^{\mathrm{s}}-\mu_{B}^{\mathrm{s}}\right) / k_{\mathrm{B}} T$.

Near the critical point, the profile obeys the scaling law: $\phi(z)=z^{-\beta_{\mathrm{t}} / \nu_{\mathrm{t}}} f\left(z / \xi_{\mathrm{t}}\right)$, where $\xi_{\mathrm{t}} \sim a\left|1-T_{\mathrm{c}} / T\right|^{-\nu_{\mathrm{t}}}$ is the thermal correlation length ( $a$ is an atomic scale) and $f(x)$ is a universal scaling function. The latter was calculated to the first order in $\epsilon=4-d$ (4 is the critical dimension) by Brézin and Leibler [8,9] using the Renormalization-Group ( $\mathrm{RG}$ ) approach. To investigate critical adsorption, these authors started with a semi-infinite space with an appropriate boundary condition incorporating the order parameter and its first derivative at the surface. In fact, this condition depends on two microscopic parameters $(c, h)$, where the coupling constant $c$ that measures the interaction strength between mixture and surface, is positive, but the surface field $h$ described above, must be strong enough $(h \rightarrow \infty)$ to ensure the condensation of the preferred species on the surface. This limit defines another surface universality class, called normal transition $[13,14,19]$. Indeed, even in the high-temperature regime $\left(T>T_{\mathrm{c}}\right)$, the non-zero value of the surface field yields a non-trivial order parameter profile. More developments using $R G$ related to the critical adsorption can be found in [10-12].

Very recently, critical adsorption was also observed experimentally for critical polymer solutions. Within this context, Craig and Law [20] accomplished ellipsometric measurements of critical adsorption choosing five solutions of polystyrene ( $A$-component) in cyclohexane ( $B$-component), for various molecular weights of the former. Measurements agree with theoretical predictions of Fisher and de Gennes [7]. Notice that the considered mixture is made of a polymer (polystyrene) and a simple liquid (cyclohexane).

Critical fluids confined between two parallel plates termed critical film [21,22], which may be a fluid near the liquid-gas critical point, a binary liquid near the consolute point, or liquid ${ }^{4} \mathrm{He}$ near the $\lambda$-point, generate long-range forces between the confining walls [21]. This force that originates from strong fluctuations of the order parameter near the critical point, is called critical Casimir force in the literature [21]. The word "Casimir" is related to the well-known Casimir effect discovered by Casimir [23], according to which the vacuum quantum fluctuations of a confined

\footnotetext{
${ }^{1}$ An extensive list of very recent experiments using some new techniques can be found in: [5]. Critical adsorption on curved objects, such as single spherical and rod-like colloidal particles, has been investigated in: [15]
} 
electromagnetic field generate an attractive force between two parallel uncharged conducting plates, which are separated by a finite distance $L$. This effect has received its final confirmation in recent experiments [24,25]. Fisher and de Gennes [7] remarked an analogous effect, arising in Statistical and Condensed Matter Physics, for the systems exhibiting a critical point and restricted by boundaries. For this case, the large-scale critical fluctuations of density appear to be the analog of the vacuum quantum fluctuations.

Near criticality, Casimir forces are universal scaling functions [26-30]. When the critical temperature is reached, these functions reduce to negative power laws in the separation $L$, which multiply universal amplitudes. Theoretically, the critical Casimir force has been extensively investigated, using several techniques. In dimension $d=2$, results were obtained using conformal invariance [31-35]. In dimension $d=3$, use was made of the field-theoretical RG [21,22,29,36-39], with the $\epsilon$-expansion procedure $(d=4-\epsilon)$. Use was also made of the Monte Carlo (MC) simulation [40]. From an experimental point of view, critical films or colloids immersed in binary liquid mixtures have been the subject of numerous experiments ${ }^{2}$ [41].

The physical system we consider in this paper is a mixture of two incompatible long polymers $A$ and $B$ dissolved in a common good solvent. We assume that the ternary polymer solution is confined between two adsorbing parallel plates 1 and 2 , separated by a distance $L$ much smaller than the thermal correlation length $\xi_{\mathrm{t}}$ $\left(L \ll \xi_{\mathrm{t}}\right)$. This characteristic length that will be defined below, measures the spatial extent of correlations. The opposite case, where $L \gg \xi_{\mathrm{t}}$, contributes to the leading critical behavior only by exponentially decreasing small corrections $[26,42]$. We assume that the plates strongly attract one of the two polymers close to the consolute point. This means that one is in the critical adsorption conditions. As a result, the plates experience an effective force resulting from strong fluctuations of the composition in the critical region, which depends on separation $L$ and the considered surface universality class. For simplicity, we will assume that either plates have the same preference to attract one component (symmetric plates) or they have an opposed preference (asymmetric plates). These two boundary conditions or surface universality classes will be denoted by: $(\uparrow \uparrow)$ or $(\uparrow \downarrow)$. Besides the chemical segregation between unlike chains, one is in the presence of excluded volume interactions leading to the swelling of chains. The question to answer is about the effect of the presence of a good solvent on the force expression.

Our findings are as follows. The first step consists in computing the induced force using the standard blob model introduced many years ago by Broseta, Leibler and Joanny [43], which is a direct consequence of renormalization theory [43-46]. In this model, chains are viewed as sequences of blobs, whose size coincides with the usual screening length $\xi$, but unlike chains interact through a Flory effective interaction parameter defined below. We show that the induced force is attractive for symmetric plates, and repulsive for asymmetric ones. For the two cases, we show that the induced forces decay like $L^{-4}$, and compute exactly the associated universal amplitudes. These are similar to the amplitudes corresponding to the molten state [47-49],

${ }^{2} \mathrm{~A}$ list of recent experimental works can be found in $[21,22]$. 
up to a multiplicative power factor of the monomer concentration. The blob model is a mean-field theory, which is reliable only for extremely high molecular-weight or very strong monomer concentration [43]. To go beyond this approximation, and in order to get a correct induced force close to the consolute point, we use a $\varphi^{4}$ field theory (the $\varphi$-field is the order parameter or composition fluctuation), to which the Renormalization-Group machineries [43-46] can be applied. This field theory is supplemented by suitable boundary conditions on the two confining plates incorporating two pairs of surface microscopic parameters $\left(c_{1}>0, h_{1}\right)$ and $\left(c_{2}>0, h_{2}\right)$. The parameters $c_{i}$ measure the interaction strengths between polymers and plates, and $h_{i}$ represents the surface chemical potential differences. These latter play the role of surface magnetic fields for magnetic materials. Critical adsorption emerges for high surface fields. For instance, the two considered surface universality classes $(\uparrow \uparrow)$ and $(\uparrow \downarrow)$ correspond to the limits $\left(h_{1} \rightarrow+\infty, h_{2} \rightarrow+\infty\right)$ and $\left(h_{1} \rightarrow+\infty, h_{2} \rightarrow-\infty\right)$, respectively. Our central result is that the total induced force is the sum of two parts. The first one is the mean-field force that decreases with the distance like $L^{-4}$, and the second one represents the force deviation originating from strong fluctuations of composition, and which decays rather as $L^{-3}$, with a known universal amplitude. This implies the existence of some cross-over distance $L^{*} \sim a N \phi^{1 / 2}$ depending on the polymerization degree $N$ of chains and the monomer fraction $\phi$, and which separates two distance-regimes. For small separations $\left(L<L^{*}\right)$, the mean-field force dominates, while for high separations $\left(L>L^{*}\right)$, the fluctuation force becomes more important.

Finally, this paper extends some recent papers on the computation of the induced force for confined critical binary polymer mixtures [47-49].

The remaining presentation proceeds as follows. Section 2 deals with the computation of the induced force for solutions of polymer blends, within the framework of mean-field theory and RG. We draw our conclusions in section 3.

\section{Computation of the induced force}

\subsection{Mean-field results}

In order to construct the free energy enabling us to compute the expected force, we start with recalling some useful background on the demixing transition in the presence of a good solvent.

Consider a mixture of two chemically different polymers $A$ and $B$, dissolved in a common good solvent. This mixture may be polystyrene (PS)-poly(methyl methacrylate) (PMMA) in toluene or PS-PDMS (poly(dimethylsiloxane)) in propylbenzene. The ternary mixture is assumed to be confined between two interacting parallel plates 1 and 2, which are separated by a finite distance $L$ smaller than the thermal correlation length $\xi_{\mathrm{t}}\left(L \ll \xi_{\mathrm{t}}\right)$. We suppose, as before, that near the critical point $T_{\mathrm{K}}$ one species has the tendency to condensate near the preferred plate. The quantities $T_{\mathrm{K}}$ and $\xi_{\mathrm{t}}$ will be defined below.

To simplify, we assume that the two polymers $A$ and $B$ have the same polymer- 
ization degree $N$. Thus, we are concerned with a monodisperse system. We denote the overall monomer fraction by $\phi=\phi_{A}+\phi_{B}$, where $\phi_{A}$ and $\phi_{B}$ are the respective monomer fractions of $A$ and $B$-polymers.

In a dilute solution, where the overall monomer fraction $\phi$ is below the threshold $\phi^{*} \sim N^{1-d \nu}(\nu \simeq 0.588$ [50] in $d=3), A$ and $B$-chains behave like separated swollen coils avoiding each other completely, and in principle no phase separation is expected. In semi-dilute solution $\left(\phi^{*} \ll \phi \ll 1\right)$, however, chains overlap and can be viewed as a sequence of uncorrelated subunits or blobs of types $A$ and $B$. Each chain contains $Z(\phi) \sim N \phi^{1 /(d \nu-1)}$ blobs. The blob size or screening length, $\xi(\phi)$, depends only on the total monomer fraction $\phi$, and scales as [51]: $\xi(\phi) \sim a \phi^{\nu /(1-\nu d)}$, where $a$ is the monomer size.

Using the renormalization theory, the authors of [43] have shown that, for a high-molecular weight solution, a given chain cannot distinguish between an $A$ and $B$-chain. This means that the chemical mismatch is irrelevant, and manifests itself only in correction to the leading behavior of the osmotic pressure. In fact, these corrections are important and govern the thermodynamics of demixing transition. From the obtained expression of the osmotic pressure, the authors derived the following free energy per site (blob)

$$
\frac{F_{0}}{k_{\mathrm{B}} T}=\frac{x}{Z(\phi)} \ln x+\frac{1-x}{Z(\phi)} \ln (1-x)+\widehat{\chi}(\phi) x(1-x),
$$

where $x=\phi_{A} / \phi$ is the composition of polymer $A$. In the above equality,

$$
\widehat{\chi}(\phi) \simeq \chi \phi^{\widetilde{\Delta}_{2}}
$$

accounts for the Flory effective interaction parameter between unlike blobs, where $\chi$ is the standard Flory interaction parameter, and $\widetilde{\Delta}_{2}$ is some crossover exponent, which in $d=3$ is given by

$$
\widetilde{\Delta}_{2} \simeq 0.30
$$

Such a value agrees with experiment ${ }^{3}$. This exponent characterizes the correction to the osmotic pressure [43]. Expression $(2 a)$ can be understood, in a certain sense, as a renormalization of interactions due to the chemical mismatch between $A$ and $B$-polymers. Typical values of the effective interaction parameter $\widehat{\chi}$ near demixing concentration are $10^{-3}$ to $10^{-2}$ for strongly incompatible pairs such as PS-PMMA of molecular weight $M_{\mathrm{w}} \sim 10^{6}$ [53], and $10^{-2}$ to $10^{-1}$ for the more incompatible pair PS-PDMS of the same molecular weight ${ }^{3}$.

Going back to expression (1), we note that it shows an obvious analogy with that defining the usual Flory-Huggins free energy of a mixture of two polymers $A$ and $B$ in the molten state $[51,52]$. The difference is that $A$ and $B$-chains have blobs of size $\xi$ as new subunits, and the segregation parameter is no longer $\chi$ but the effective one $\widehat{\chi}$ defined by relations $(2 a)$ and $(2 b)$. Of course, these two parameters coincide in the limit $\phi \rightarrow 1$.

\footnotetext{
${ }^{3}$ See [43] and experimental references therein.
} 
The model of free energy defined through relations (1), (2a) and (2b) constitutes the so-called blob model [43], which is a direct consequence of renormalization theory.

Let us recall the analytical expression for the demixing critical point location, which can be obtained by equating to zero the first and the second derivatives of free energy (1) with respect to composition $x$. One gets the location of the critical point [43]

$$
\widehat{\chi}\left(\phi_{\mathrm{K}}, T\right)=\frac{2}{Z(\phi)}, \quad x_{\mathrm{K}}=\frac{1}{2}
$$

where $\phi_{\mathrm{K}}$ is the critical monomer fraction whose expression can be found in [43]. We simply note that $\phi_{\mathrm{K}}$ is larger than the overlap monomer fraction $\phi^{*}$ defined above. The critical point is located at the top of the coexistence curve. Below $\phi_{\mathrm{K}}\left(\phi<\phi_{\mathrm{K}}\right)$, the ternary mixture is homogeneous, while above $\phi_{\mathrm{K}}\left(\phi>\phi_{\mathrm{K}}\right)$, this mixture phase separates in two phases alternatively rich in $A$ and $B$-polymers. Finally, we recall that the critical temperature $T_{\mathrm{K}}$, at a fixed concentration, is given by [43]

$$
\chi\left(T_{\mathrm{K}}\right) \simeq \phi^{-1 / b} N^{-1},
$$

with the exponent $b \simeq 0.62(d=3)$ [43]. The above relation tells us that the critical temperature $T_{\mathrm{K}}$ should be proportional to the polymerization degree $N$.

Now, to describe the critical phase behavior of the ternary mixture, we introduce an order parameter that is defined by

$$
x=\frac{1+\widehat{x}}{2}
$$

where $x$ is the composition of species $A$. The above definition means that the order parameter $\widehat{x}$ is proportional to the shift $x-x_{\mathrm{K}}$, where $x_{\mathrm{K}}=1 / 2$ is the critical composition. The order parameter $\widehat{x}$ depends on the $d$-dimensional position vector $\mathbf{r}=(\rho, z)$, where $\rho \in \mathbf{R}^{d-1}$ is the transverse vector and $z \in[0, L]$ is the perpendicular distance from plate 1 taken as the origin. Thus, the two plates (hyperplanes) 1 and 2 are located at $z=0$ and $L$, respectively. The homogeneity property of plates implies that $\widehat{x}$ depends only on the perpendicular distance $z$. We denote by $\widehat{x}_{1}$ and $\widehat{x}_{2}$ the respective values of the order parameter on plates 1 and 2. Symmetric plates correspond to $\widehat{x}_{1}=\widehat{x}_{2}$, and asymmetric ones correspond to $\widehat{x}_{1}=-\widehat{x}_{2}$. Since swollen $A$ and $B$-chains can be regarded as sequences of new subunits or blobs, but interact chemically through the Flory effective interaction parameter $\widehat{\chi}$ defined by equations $(2 a)$ and $(2 b)$, the total free energy (per unit area) is given by a formula similar to that defining a binary polymer mixture [49]. Then, we write

$\frac{F_{0}[\widehat{x}]}{\mathcal{A} k_{\mathrm{B}} T}=\sum_{i=1,2}\left(-h_{i}^{0} \widehat{x}_{i}+\frac{c_{i}^{0}}{2} \widehat{x}_{i}^{2}\right)+\xi^{-d}(\phi) \int_{0}^{L} \mathrm{~d} z\left[\frac{t_{0}}{2} \widehat{x}^{2}+\frac{u_{0}}{4} \widehat{x}^{4}+\kappa(\phi)\left(\frac{\mathrm{d} \widehat{x}}{\mathrm{~d} z}\right)^{2}\right]$

where $\mathcal{A}$ is the common area of plates. Here, $t_{0}=(2 / Z(\phi)-\widehat{\chi}) / 2$ is the reduced temperature, $u_{0}=1 / 3 Z(\phi)$ is the coupling constant, $\kappa(\phi)=\xi^{2}(\phi) / 9$, and $\left(c_{i}^{0}, h_{i}^{0}\right)$ are the surface microscopic parameters relative to plates 1 and 2 . Notice that the 
integrand in the bulk part of the above free energy can be obtained expanding the free energy (1) to the fourth order around the critical composition $x_{\mathrm{K}}=1 / 2$. The gradient term is introduced to take into account the interfacial energy between $A$ and $B$-rich phases.

We note that the above free energy is similar to that corresponding to a confined binary polymer mixture [49], with the simple substitutions: $a \rightarrow \xi, N \rightarrow Z(\phi)$. This means that chains in the solution can be regarded as sequences of $Z(\phi)$ blobs of the same size $\xi$. Taking advantage of the results in [49] and using the above substitutions, we find for the induced forces (per unit area)

$$
\frac{\Pi_{a}^{0}}{k_{\mathrm{B}} T_{\mathrm{K}}}=\frac{\Delta_{\uparrow \uparrow}^{0}}{L^{4}}
$$

for symmetric (or attractive) plates, and

$$
\frac{\Pi_{r}^{0}}{k_{\mathrm{B}} T_{\mathrm{K}}}=\frac{\Delta_{\uparrow \downarrow}^{0}}{L^{4}}
$$

for asymmetric (or repulsive) ones, with the following universal amplitudes

$$
\begin{aligned}
& \Delta_{\uparrow \uparrow}^{0}=-a N \frac{[\boldsymbol{\Gamma}(1 / 4)]^{8}}{432 \pi^{2}} \phi^{(1-\nu) /(3 \nu-1)}, \\
& \Delta_{\uparrow \downarrow}^{0}=N a \frac{[\boldsymbol{\Gamma}(1 / 4)]^{8}}{108 \pi^{2}} \phi^{(1-\nu) /(3 \nu-1)} .
\end{aligned}
$$

Here, $\boldsymbol{\Gamma}(x)$ is the gamma function [53].

Let us comment on these results.

Firstly, we note that the above expressions obtained within the framework of the blob model show that the presence of a good solvent simply induces a renormalization of the force amplitudes, through the multiplicative power factor $\phi^{(1-\nu) /(3 \nu-1)} \sim$ $\phi^{1 / 2}(d=3, \nu=3 / 5)$ depending on the monomer fraction $\phi$.

Secondly, for both symmetric and asymmetric plates, the attractive and repulsive forces decay according to the same negative fourth power law, but with different amplitudes.

Thirdly, as for confined polymer blends, the repulsive force is four times more important than the attractive one. The reason for that is explained in [49].

Finally, in the limit $\phi \rightarrow 1$, we recover the results corresponding to the molten state [49].

The blob model is a mean-field theory, and it was found [43] that this is valid only for an extremely high molecular-weight or a very high monomer concentration. To go beyond the mean-field theory, and in order to obtain correct results close to the critical point where fluctuations of composition are strong enough, we shall use the renormalization theory applied to the field theory described below. 


\section{2. $R G$ results}

The first step consists in rewriting the above free energy (6) by rescaling the composition fluctuations in bulk and at the surfaces and parameters of the problem, according to

$$
\begin{aligned}
\varphi=\sqrt{\kappa(\phi)}[\xi(\phi)]^{-d / 2} \widehat{x}, & \varphi_{i} & =\sqrt{\kappa(\phi)}[\xi(\phi)]^{-d / 2} \widehat{x}_{i}, \\
t=\frac{1}{\kappa(\phi)} t_{0}, & g & =\frac{6}{[\kappa(\phi)]^{2}}[\xi(\phi)]^{d} u_{0}, \\
c_{i}=\frac{1}{\kappa(\phi)}[\xi(\phi)]^{d} c_{i}^{0}, & h_{i} & =\frac{1}{\sqrt{\kappa(\phi)}}[\xi(\phi)]^{d / 2} h_{i}^{0} .
\end{aligned}
$$

Here, $\left(t_{0}, u_{0}\right)$ and $\left(c_{i}^{0}, h_{i}^{0}\right)$ are the parameters defined above, where $\xi(\phi)$ is the screening length.

With these considerations, the total free energy rewrites

$$
\frac{F[\varphi]}{k_{\mathrm{B}} T}=\sum_{i=1,2} \int \mathrm{d} \rho\left(-h_{i} \varphi_{i}+\frac{c_{i}}{2} \varphi_{i}^{2}\right)+\int \mathrm{d} \mathbf{r}\left[\frac{1}{2}(\nabla \varphi)^{2}+\frac{t}{2} \varphi^{2}+\frac{g}{4 !} \varphi^{4}\right]
$$

The $\varphi$-field depends on the spatial coordinates $\mathbf{r}=(\rho, z)$, with $\rho \in \mathbf{R}^{d-1}$ and $0 \leqslant z \leqslant L, \varphi_{i}$ being the surface fields defined on the $(d-1)$-dimensional plates 1 and 2. $t \sim\left(T-T_{\mathrm{K}}\right) / T_{\mathrm{K}}$ represents the reduced temperature, $g$ is the coupling constant, and $\left(c_{i}, h_{i}\right)$ are the new surface parameters. Then, fields $\varphi$ and $\varphi_{i}$, and bulk and surface parameters have the following dimensions: $[\varphi]=l^{1-d / 2},[\varphi]=\left[\varphi_{i}\right]=l^{1-d / 2}$, $[t]=l^{-2},[g]=l^{d-4},\left[c_{i}\right]=l^{-1}\left(c_{i}^{-1}\right.$ is the extrapolation length $),\left[h_{i}\right]=l^{-d / 2}$, where $l$ is some length. At the critical dimension of the system $d_{\mathrm{c}}=4$, the coupling constant $g$ becomes marginal.

Thus, our theoretical model is a $\varphi^{4}$-field theory described by the above LandauGinzburg-Wilson Hamiltonian. Recall that critical adsorption emerges in the limit $h_{i} \rightarrow \pm \infty$.

The second step consists in computing the Casimir force using this field theory. We first note that the above Hamiltonian is nothing else but that describing the critical properties of binary liquid mixtures of small molecules near the consolute point, one-component fluids near the liquid-gas critical point, or Ising-like magnetic materials near the Curie temperature. Thus, the $\varphi$-field (order parameter) may play the role of the difference between the compositions for simple liquid mixtures, the difference between liquid and gas densities for one-component fluids, or the local magnetization for Ising-like magnetic materials. In this sense, the ternary mixture of our interest belongs to the universality class $(n=1, d)$, where $n$ is the number of components of the order parameter. Hence, the critical phase behavior for ternary polymer mixtures is of Ising type [17,18]. As noted in [43], this fact seems to be in good agreement with the recent light scattering experiments ${ }^{3}$, essentially based on the so-called "optical $\theta$ " method [54]. For instance, in a recent experiment [55], 
one has studied the solutions of PS and PDMS in propylbenzene, and found that coexistence curve exponent $\beta_{\mathrm{t}}$ is close to the Ising theoretical value.

We can thus take advantage of the work by Krech [29], which is concerned with the computation of the Casimir force in confined liquid mixtures. To determine the force expression for confined ternary polymer mixtures, we shall follow the techniques used by the author.

Let us first write the Casimir force as

$$
\Pi_{a, r}=\Pi_{a, r}^{0}+\delta \Pi_{a, r}
$$

where the quantity $\Pi_{a, r}^{0}$ represents the mean-field force calculated above, relations (7) or (8). The remaining part, $\delta \Pi_{a, r}$, accounts for the force deviation due to strong fluctuations of the composition. We recall that the induced force is defined through the expectation mean-value of perpendicular component of the stress tensor [29], which has been calculated using the so-called loop expansion $[17,18]$. The meanfield contribution $\Pi_{a, r}^{0}$ represents the zeroth order of this expansion, while $\delta \Pi_{a, r}$ accounts for the contribution of higher orders.

To determinate the force deviation $\delta \Pi_{a, r}$, we start from the Casimir free energy per unit area, $\delta f_{a, r}$, resulting from fluctuations of the composition. According to [29], this free energy can be written as

$$
\frac{\delta f_{a, r}}{k_{\mathrm{B}} T}=\frac{1}{L^{d-1}} g_{a, r}\left(\frac{L}{\xi_{\mathrm{t}}}\right)
$$

In this scaling form, the factor $1 / L^{d-1}$ simply expresses the natural dimension of the reduced Casimir energy $\delta f_{a, r} / k_{\mathrm{B}} T$. Here, $\xi_{\mathrm{t}} \sim R(\phi)[Z(\phi)]^{-\nu_{\mathrm{t}}+1 / 2}\left|1-T / T_{\mathrm{K}}\right|^{-\nu_{\mathrm{t}}}$ is the thermal correlation length, where $\nu_{\mathrm{t}} \simeq 0.63$ is the standard Ising exponent, and $R(\phi) \sim a N^{1 / 2} \phi^{(2 \nu-1) / 2(1-3 \nu)}$ represents the size of a chain in semi-dilute solution [51], with the swelling exponent $\nu \simeq 0.588$ ([50]) that must not be confused with $\nu_{\mathrm{t}}$. On the other hand, the scaling function $g_{a, r}(x)$ is analytic for $x \ll 1$ $\left(L \ll \xi_{\mathrm{t}}\right)$. Then, at the critical point $T=T_{\mathrm{K}}\left(\xi_{\mathrm{t}} \rightarrow \infty\right), g_{a, r}(0)$ is finite and we write it as: $g_{a}(0)=\delta \Delta_{\uparrow \uparrow} /(d-1)$ or $g_{r}(0)=\delta \Delta_{\uparrow \downarrow} /(d-1)$, where $\delta \Delta_{\uparrow \uparrow}$ and $\delta \Delta_{\uparrow \downarrow}$ are the Casimir amplitudes (in our notations). With these considerations, at criticality, the Casimir energy decays in a universal way as

$$
\frac{\delta f_{a}}{k_{\mathrm{B}} T_{\mathrm{K}}}=\frac{\delta \Delta_{\uparrow \uparrow} /(d-1)}{L^{d-1}}, \quad \frac{\delta f_{r}}{k_{\mathrm{B}} T_{\mathrm{K}}}=\frac{\delta \Delta_{\uparrow \downarrow} /(d-1)}{L^{d-1}} .
$$

The Casimir force deviation (per unit area), $\delta \Pi_{a, r}$, is just minus the first derivative of $\delta f_{a, r}$, with respect to separation $L: \delta \Pi_{a, r}=-\partial \delta f_{a, r} / \partial L$. We then find at three dimensions

$$
\frac{\delta \Pi_{a}}{k_{\mathrm{B}} T_{\mathrm{K}}}=\frac{\delta \Delta_{\uparrow \uparrow}}{L^{3}}
$$

for attractive walls, and

$$
\frac{\delta \Pi_{r}}{k_{\mathrm{B}} T_{\mathrm{K}}}=\frac{\delta \Delta_{\uparrow \downarrow}}{L^{3}}
$$


for repulsive ones.

We note that, in general, the force amplitudes are universal, and they depend only on space dimension $d$ and surface universality class (the choice of boundary conditions). The amplitudes $\delta \Delta_{\uparrow \uparrow}$ and $\delta \Delta_{\uparrow \downarrow}$ have been calculated through a perturbative expansion with respect to the coupling constant $g$. Then, at a fixed point $g^{*}$, these amplitudes become a series in $\epsilon=4-d$ ( 4 is the critical dimension of the system) that must be resummed using Borel-Leroy techniques ${ }^{4}$ to get their best values at dimension $d=3(\epsilon=1)$. All these questions have been addressed in [29], and we simply give the values of these force amplitudes in $d=3$

$$
\delta \Delta_{\uparrow \uparrow} \simeq-0.652, \quad \delta \Delta_{\uparrow \downarrow} \simeq 4.48 .
$$

These values are in good agreement with MC simulation [56].

With these considerations, the total Casimir forces (per unit area) write

$$
\begin{aligned}
\frac{\Pi_{a}}{k_{\mathrm{B}} T_{\mathrm{K}}} & =\frac{\Delta_{\uparrow \uparrow}^{0}}{L^{4}}+\frac{\delta \Delta_{\uparrow \uparrow}}{L^{3}}, \\
\frac{\Pi_{r}}{k_{\mathrm{B}} T_{\mathrm{K}}} & =\frac{\Delta_{\uparrow \downarrow}^{0}}{L^{4}}+\frac{\delta \Delta_{\uparrow \downarrow}}{L^{3}},
\end{aligned}
$$

where the mean-field amplitudes $\Delta_{\uparrow \uparrow}^{0}$ and $\Delta_{\uparrow \downarrow}^{0}$ are defined by equations (7a) and (8a).

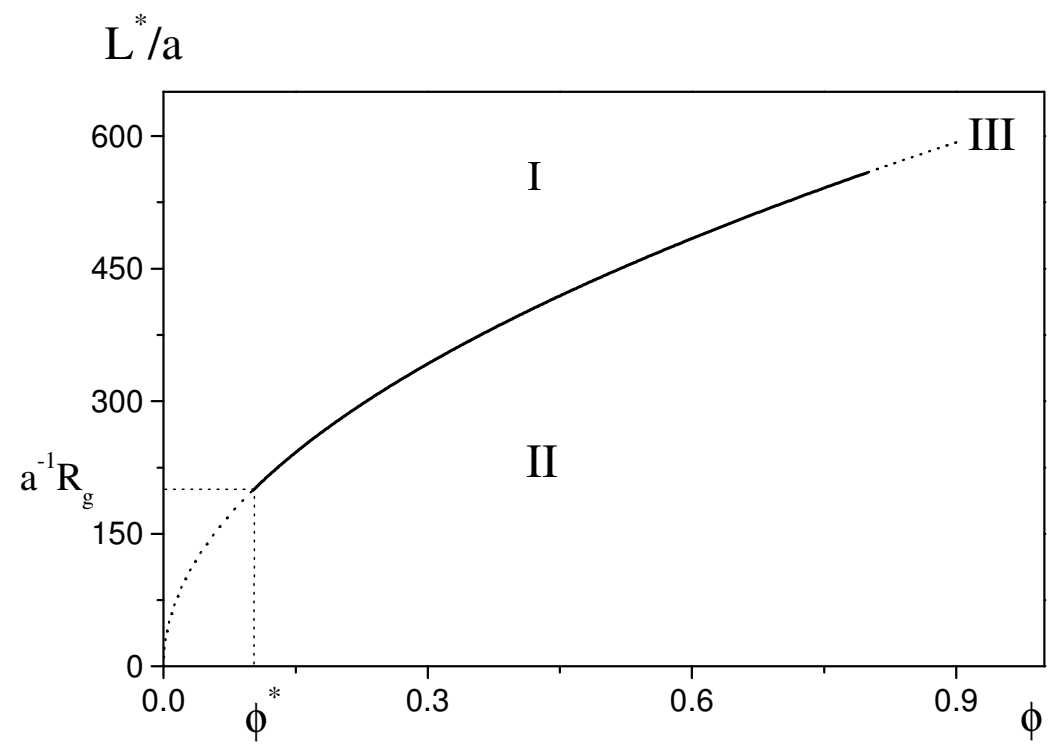

Figure 1. Cross-over curve III separating the two domains I and II in the $(\phi, L)$ plane. In the former (high distance-regime), the fluctuations force dominates, and in the second one (small distance-regime), the mean-field force is more important. This curve is drawn with parameter $N=100$.

These results call for some comments.

\footnotetext{
${ }^{4}$ See, for instance, Refs. [17] and [18].
} 
Firstly, equations (17) and (18) tell us that, when they are reduced by the $k_{\mathrm{B}} T_{\mathrm{K}^{-}}$ factor, the attractive and repulsive Casimir forces $\Pi_{a, r}$ are universal, independently of the chemical structure of polymers and plates.

Secondly, we emphasize that the force expressions (17) and (18), when they are compared to those corresponding to the molten state [49], indicate that the solvent induces a drastic change of the force expression. Indeed, the swelling of chains modifies the dependence of the force on the distance, through the appearance of the $L^{-3}$-decay. This change of the behavior is not surprising, since in the presence of a good solvent, fluctuations of composition close to the consolute point are strong enough.

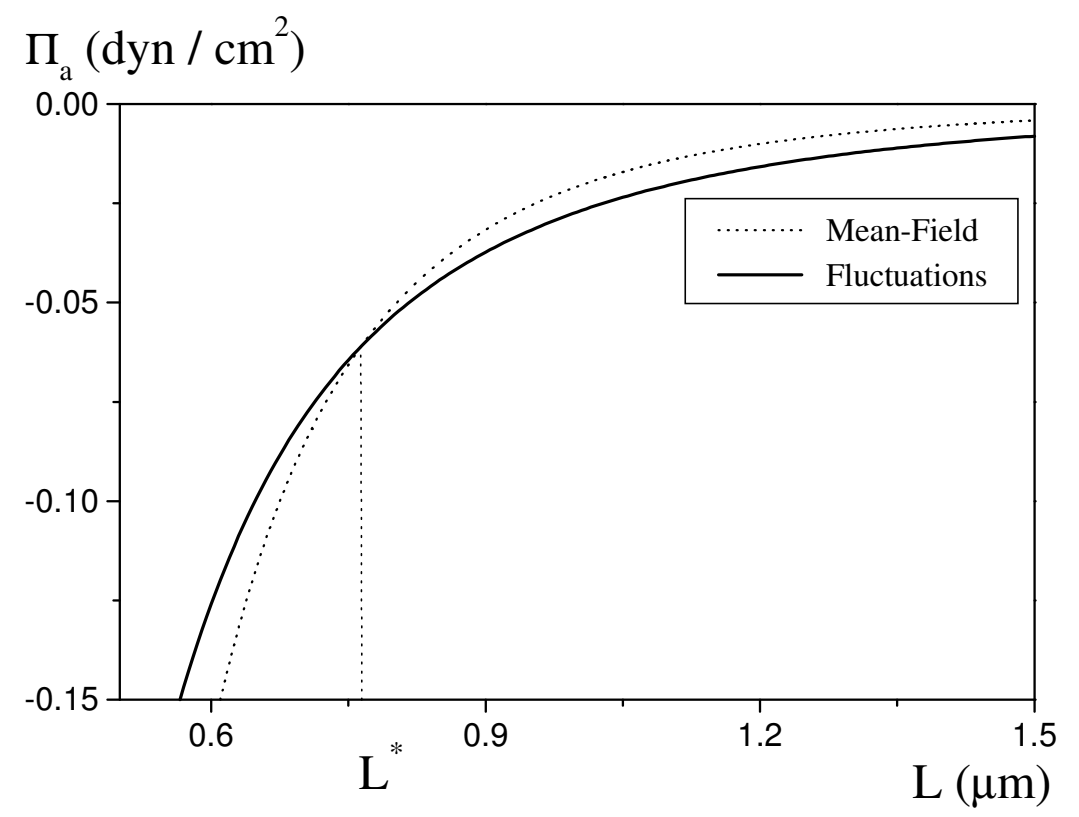

Figure 2. Superposition of curves representing attractive mean-field force (dashed line) and attractive fluctuation force (solid line), versus separation $L$. Curves are drawn with parameters: $a=10$ Angstroms, $N=100, \phi=0.5$.

Thirdly, the above formulae suggest the existence of a cross-over phenomenon occurring at some characteristic distance $L^{*}$, obtained by making a comparison between the mean-field contribution $\left(\sim L^{-4}\right)$ and the fluctuation one $\left(\sim L^{-3}\right)$. This comparison gives the cross-over distance

$$
L^{*} \sim a N \phi^{(1-\nu) /(3 \nu-1)} \sim a N \phi^{1 / 2},
$$

which depends on the molecular-weight (through $N$ ) and monomer fraction $\phi$. At the threshold $\phi \sim \phi^{*}$, the length $L^{*}$ becomes of the order of the gyration radius $R_{\mathrm{G}} \sim a N^{\nu}$ of a single chain in a dilute solution. Therefore, this defines, at a fixed molecular-weight, a cross-over line III separating two domains I and II in the $(\phi, L)$ plane (figure 1). In the high separation domain $\left(L>L^{*}\right)$, fluctuations of composition dominate, and then, the effective force behaves as $L^{-3}$. In low separation domain II $\left(L<L^{*}\right)$, however, a mean-field result is expected, and the effective force scales 


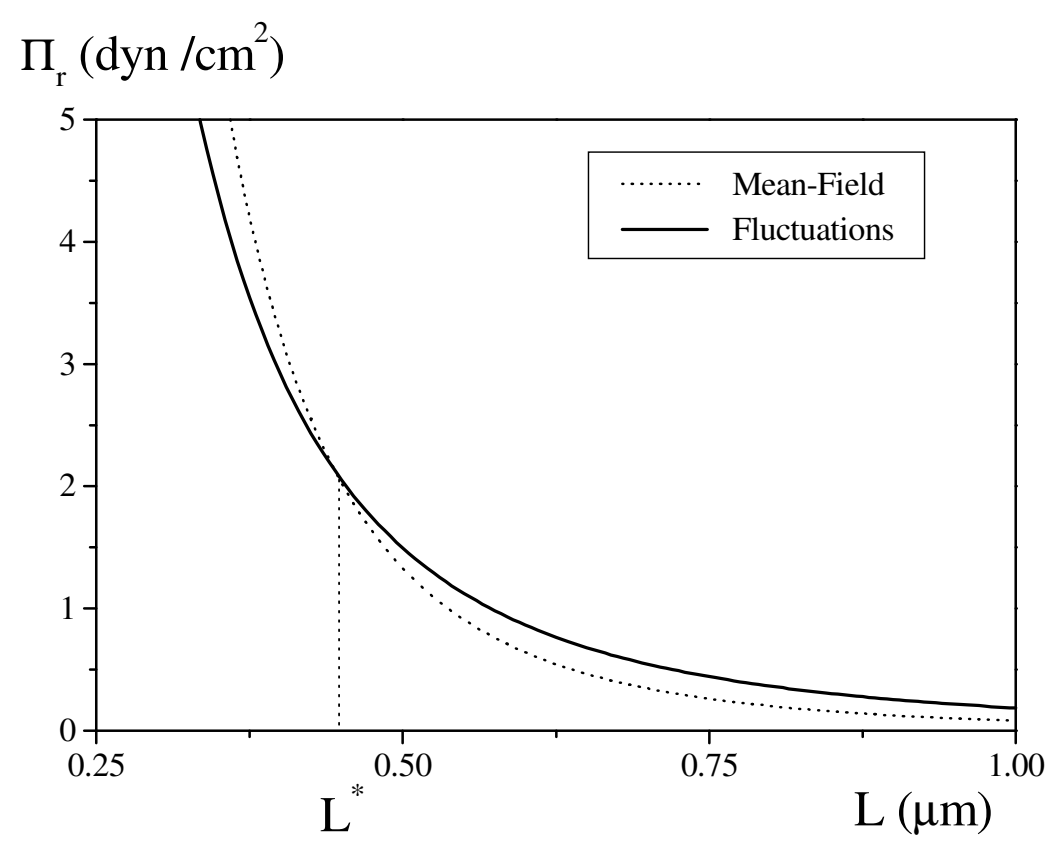

Figure 3. Superposition of curves describing repulsive mean-field force (dashed line) and repulsive fluctuation force (solid line), versus separation $L$. Curves are drawn with parameters: $a=10$ Angstroms, $N=100, \phi=0.5$.

rather as $L^{-4}$. Indeed, this can be understood as follows. When the distance between the plates is lowered, the local monomer concentration is increased, resulting in strong screening of excluded volume interactions. That is why the mean-field theory works at small distances.

In figure 2, we superpose the curves representing an attractive mean-field $\Pi_{a}^{0}$ force (dashed line) and attractive fluctuation force $\delta \Pi_{a}$ (solid line), versus separation $L$. In figure 3, we report the curves describing repulsive mean-field $\Pi_{r}^{0}$ force (dashed line) and repulsive fluctuation force $\delta \Pi_{r}$ (solid line), versus separation $L$. For the two cases, the curves intersect at the cross-over distance $L^{*}$, which is different for the two boundary conditions. All these curves are drawn with parameters: $a=10$ Angstroms, $N=100, \phi=0.5$.

\section{Conclusions}

The purpose of the present work is to determine the Casimir force within the confined ternary polymer solutions between two parallel adsorbing plates. These solutions are made of two incompatible polymers $A$ and $B$ dissolved in a common good solvent. In addition to the chemical segregation between unlike chains, excluded volume interactions are present.

To compute the expected force, we have restricted ourselves to two surface universality classes: symmetric and asymmetric plates. The induced force is attractive for symmetric plates, and repulsive for asymmetric ones. Calculations were done, first, using the blob model. For the two boundary conditions, we have shown that 
the forces decay similarly to $L^{-4}$. We found that the force amplitudes are similar to those corresponding to the molten state, up to a multiplicative power factor of the monomer concentration.

The blob model is a mean-field theory, which is valid only for very strong monomer concentrations or extremely high molecular-weights. To see this, denote by $\Delta^{*} \phi=\phi-\phi_{\mathrm{K}}$ and $\Delta^{*} T=T-T_{\mathrm{K}}$, respectively, the range of monomer concentrations and temperatures, for which the fluctuations of the composition are strong enough, so that the mean-field approach is no longer reliable. The size of the critical region has been determined using a Ginzburg criterion [43]. We simply sketch the result that: $\Delta^{*} \phi / \phi_{\mathrm{K}} \sim M^{-\Delta_{2} /\left(1+\Delta_{2}\right)}$, and $\Delta^{*} T / T_{\mathrm{K}} \sim\left(\phi_{\mathrm{K}} / \phi^{*}\right)^{-1 /(3 \nu-1)}$, where $M$ is the molecular-weight and $\Delta_{2}=\widetilde{\Delta}_{2}(3 \nu-1) \simeq 0.22$ is a cross-over exponent. Thus, in the limit of extremely long chains and very high concentrations, the above expressions suggest that the critical region is very narrow, and then, the phase behavior can be obtained using the mean-field approximation. A typical value of the molecular-weight may be $M=2.2 \times 10^{6}$ for the nearly pair PS-PMMA [57].

To go beyond the blob model, and in order to obtain a correct expression for the induced force, we applied the RG-machineries to a $\varphi^{4}$-field theory we described above. We have shown the existence of two distance-regimes. Below some characteristic length $L^{*} \sim a N \phi^{1 / 2}$, mean-field theory can be applied, an then, the force decays as $L^{-4}$. Above $L^{*}$, however, one assists to a drastic change of the force expression due to the presence of strong fluctuations of the composition. In this regime, it was found that the force decreases rather as $L^{-3}$.

We point out that this paper is a natural extension of the recent published ones, which were concerned with the computation of the induced force for confined critical binary polymer mixtures. The difference between these papers and the present one comes from the inclusion of a good solvent as a third component. This implies a change of the power-law decay of the expected force in comparison with polymer blends.

At the experimental level, we think that the induced force could be measured in an experiment similar to that used for the measurements of the repulsive force between two plates coated by adsorbed polymers [58,59], keeping fixed both the molecular-weight and the monomer concentration (above the threshold), and varying the separation between confining walls.

Finally, we emphasize that the present work can be extended to those mixtures in contact with other geometries, namely spherical colloids, or spherical colloids near a plane surface. These investigations are in progress.

\section{Acknowledgements}

We would like to thank Professor Daoud for helpful discussions. We are much indebted to Professors M. Krech and S. Dietrich for useful correspondences. We are grateful to our referee for his pertinent remarks and useful suggestions. 


\section{References}

1. Beysens D., Leibler S. // J. Phys. Lett. (Paris), 1982, No. 43, p. L-133.

2. Franck C., Schnatterly S.E. // Phys. Rev. Lett., 1982, No. 48, p. 763.

3. Schlossman M., Wu X.-L., Franck C. // Phys. Rev. B, 1985, No. 31, p. 1478.

4. Dixon J.A., Schlossman M., Wu X.-L., Franck C. // Phys. Rev. B, 1985, No. 31, p. 1509.

5. Hanke A., Krech M., Schlesener F., Dietrich S. // Phys. Rev. E, 1999, No. 60, p. 5163.

6. Blümel S., Findenegg G.H. // Phys. Rev. Lett., 1985, No. 54, p. 447.

7. Fisher M.E., de Gennes P.-G. // C. R. Acad. Sci. (Paris) Sér. B, 1978, No. 287, p. 207; de Gennes P.-G. // C. R. Acad. Sci. (Paris) II, 1981, No. 292, p. 701.

8. Brézin E., Leibler S. // Phys. Rev. B, 1983, No. 27, p. 594.

9. Leibler S. Thesis. Paris XI University, 1984.

10. Rudnik J., Jasnow D. // Phys. Rev. Lett., 1982, No. 48, p. 1059.

11. Leibler S., Peliti L. // J. Phys. C, 1982, No. 15, p. L-403.

12. Peliti L., Leibler S. // J. Phys. C, 1983, No. 16, p. L-2635.

13. Diehl H.W. Phase Transitions and Critical Phenomena, vol. 10, edited by C. Domb and J.L. Lebowitz. London, Academic Press, 1986.

14. Dietrich S. Phase Transitions and Critical Phenomena, vol. 12, edited by C. Domb and J.L. Lebowitz. London, Academic Press, 1988.

15. Hanke A., Dietrich S. // Phys. Rev. E, 1999, No. 59, p. 5081;

Hanke A. // Phys. Rev. Lett., 2000, No. 84, p. 2180.

16. Blümel S., Findenegg G.H. // Phys. Rev. Lett., 1985, No. 54, p. 447.

17. Zinn-Justin J. Quantum Field Theory and Critical Phenomena. Oxford, Clarendon Press, 1989.

18. Itzykson C., Drouffe J.M. Statistical Field Theory: 1 and 2. Cambridge University Press, 1989.

19. Binder K. Phase Transitions and Critical Phenomena, vol. 8, edited by C. Domb and J.L. Lebowitz. London, Academic Press, 1983.

20. Craig C.L., Law B.M. // J. Chem. Phys., 1996, No. 104, p. 2070.

21. Krech M. The Casimir Effect in Critical Systems. Singapore, World Scientific, 1994.

22. Krech M., Dietrich S. // Phys. Rev. Lett., 1991, No. 66, p. 345;

Krech M., Dietrich S. // Phys. Rev. Lett., 1991, No. 67, p. 1055.

23. Casimir H.B.G. // Proc. Kon. Ned. Akad. Wetenschap B, 1948, No. 51, p. 793.

An extensive list of recent works on the subject can be found in: J. Feinberg, A. Mann, and M. Revzen, hep-th/9908149, June 2000.

24. Lamoreaux S.K. // Phys. Rev. Lett., 1997, No. 78, p. 5.

25. Mohideen U., Roy A. // Phys. Rev. Lett., 1998, No. 81, p. 4549.

26. Barber N.M. Phase Transitions and Critical Phenomena, vol. 8, edited by C Domb and J.L. Lebowitz. New York, Academic Press, 1983.

27. Privman V. Finite Size Scaling and Numerical Simulation of Statistical Systems, edited by V. Privman. Singapore, World Scientific, 1990.

28. Krech M., Dietrich S. // Phys. Rev. A, 1992, No. 46, p. 1922.

29. Krech M. // Phys. Rev. E, 1997, No. 56, p. 1642.

30. Blöte H.W.J., Cardy J.L., Nightingale M.P. // Phys. Rev. Lett., 1986, No. 56, p. 742.

31. Affleck I. // Phys. Rev. Lett., 1986, No. 56, p. 746.

32. Cardy J.L. // Nucl. Phys. B, 1986, No. 275, p. 200. 
33. Burkhardt T.W., Xue T. // Phys. Rev. Lett., 1991, No. 66, p. 895;

Burkhardt T.W., Xue T. // Nucl. Phys. B, 1991, No. 345, p. 653.

34. Burkhardt T.W., Eisenriegler E. // Nucl. Phys. B, 1994, No. 424 [FS], p. 487.

35. Symanzik K. // Nucl. Phys. B, 1981, No. 190 [FS], p. 1.

36. Nightingale M.P., Indekeu J.O. // Phys. Rev. Lett., 1985, No. 54, p. 1824;

Indekeu J.O., Nightingale M.P., Wang W.V. // Phys. Rev. B., 1986, No. 34, p. 330.

37. Burkhardt T.W., Stapper M. // Phys. Rev. B, 1994, No. 50, p. 10009.

38. Burkhardt T.W., Eisenriegler E. // Phys. Rev. Lett., 1995, No. 74, p. 3189.

39. Eisenriegler E., Ritschel U. // Phys. Rev. B, 1995, No. 51, p. 13717.

40. Mon K.K. // Phys. Rev. Lett., 1985, No. 54, p. 2671.

41. Schlesener F., Hanke A., Dietrich S. // J. Stat. Phys., 2003, No. 110, p. 981;

42. Brézin E. // J. Phys. (Paris), 1982, No. 43, p. 15.

43. Broseta D., Leibler L., Joanny J.-F. // Macromolecules, 1987, No. 20, p. 1935.

44. Joanny J.F., Leibler L., Ball R. // J. Chem. Phys., 1984, No. 81, p. 4640.

45. Kosmas M.K. // J. Phys. Lett. (Paris), 1984, No. 45, p. L-889.

46. Schäfer L., Kappeler Ch. // J. Phys. (Paris), 1985, No. 46, p. 1853;

Witten T.A., Schäfer L. // J. Phys. A, 1978, No. 11, p. 1843.

47. Cherrabi R., Saout Elhak A., Benhamou M., Daoud M.

// J. Chem. Phys., 1999, No. 111, p. 8174.

48. Cherrabi R., Saout Elhak A., Benhamou M., Daoud M.

// Phys. Rev. E, 2000, No. 62, p. 6795.

49. Hachem E.-K., Benhamou M., Daoud M. // J. Chem. Phys., 2002, No. 116, p. 8168.

50. des Cloizeaux J., Jannink G. Polymers in Solution. Oxford, Oxford University Press, 1990.

51. de Gennes P.G. Scaling Concept in Polymer Physics. Ithaca, Cornell University Press, 1979.

52. Flory P.J. Principles of Polymer Chemistry. Ithaca, Cornell University Press 1953.

53. Gradshteyn I.S., Ryzik I.M. Table of Integrals, Series and Products. New York, Academic Press, 1980.

54. Tanaka T., Inagaki H. // Macromolecules, 1979, No. 12, p. 1229.

55. Shinozaki K., Saito Y., Nose T. // Polymer, 1982, No. 23, p. 1937.

56. Krech M., Landau D.P. // Phys. Rev. E, 1996, No. 53, p. 4414.

57. Fukuda T., Nagata M., Inagaki H. // Macromolecules, 1984, No. 17, p. 548.

58. Cain F., Ottewill R., Smittam J. // Faraday Discuss. Chem. Soc., 1978, No. 65, p. 33.

59. Klein J. // Nature (London), 1980, No. 228, p. 248. 


\title{
Сила Казимира в критичних трикомпонентних розчинах полімерів
}

\author{
Х.Рідоуане, Е.-К.Хачем, М.Бенхамоу \\ Лабораторія фізики полімерів та критичних явищ, \\ Факультет природничих наук Бен М'сік, В.Р. 7955 \\ Марокко, Касабланка
}

Отримано 24 листопада 2003 р.

Розглядається суміш двох несумісних полімерів $A$ i $B$, що добре розчиняються в спільному розчиннику, вміщена між двома паралельними пластинами, розділеними скінченною відстанню $L$. Ми вважаємо, що поблизу точки розчинення вони сильно притягають один з двох полімерів (критична адсорбція). При цьому пластини знаходяться під впливом ефективної сили, породженої сильними флуктуаціями суміші. Для спрощення ми припускаємо, що або обидві пластини притягають той самий компонент (симетричні пластини) або вони віддають перевагу різним компонентам (асиметричні пластини). Симетричним пластинам відповідає сила притягання, асиметричним - відштовхування. Спершу ми точно розрахували цю силу, використовуючи краплинну модель, і встановили, що сили притягання і відштовхування загасають подібним чином як $L^{-4}$. Щоб вийти поза межі краплинної моделі, яка відповідає наближенню середнього поля, і з метою отримати правильний вигляд індукованої сили, ми застосували ренорм-груповий підхід до теорії поля $\varphi^{4}(\varphi$ - флуктуація суміші) з двома відповідними граничними умовами на поверхнях. У результаті встановлено, що шукана сила є сумою двох вкладів. Перший з них - це вклад середнього поля, що загасає як $L^{-4}$, а другий - відхилення, викликане сильними флуктуаціями суміші, що зменшується радше як $L^{-3}$. Це означає, що існує певна відстань кроссоверу $L^{*} \sim a N \phi^{1 / 2}$ ( $a$ - розмір мономера, $N$ - ступінь полімеризації ланцюжків і $\phi-$ об'ємна частка мономера), що розділяє характерні відстані двох згаданих режимів. На малих відстанях $\left(L<L^{*}\right)$ переважає вплив середнього поля, тоді як для великих $\left(L>L^{*}\right)$ більш важливим стає флуктуаційний вклад.

Ключові слова: трикомпонентні розчини полімерів, обмеження, сила Казимира

PACS: $64.75 .+g, 68.45 .-v, 61.41 .+e$ 